ARTIGO ORIGINAL

\title{
Produtividade, adaptabilidade e estabilidade genotípica de clones de Eucalyptus spp. e Corymbia spp. em diferentes espaçamentos de plantio
}

\author{
Productivity, adaptability and genotypic stability of clones of Eucalyptus \\ spp. and Corymbia spp. in different plant spacing
}

Lucas Guilherme Moura Oliveira $^{1}$ (D), Ariadne Marques ${ }^{1}$ (D), Emerson Delano Lopes $^{2}$ (D), Janaína Fernandes Gonçalves ${ }^{1}$ (D), Nivaldo de Souza Martins ${ }^{3}$ (D), Claudilene Aparecida Alves Pena ${ }^{3}$ (D), David Calaes Arbex ${ }^{3}$ (D), Marcelo Luiz de Laia' ${ }^{1}$ (D)

${ }^{1}$ Universidade Federal dos Vales do Jequitinhonha e Mucuri - UFVJM, Diamantina, MG, Brasil

${ }^{2}$ Instituto Federal do Norte de Minas Gerais - IFNMG, Diamantina, MG, Brasil

${ }^{3}$ Aperam Bioenergia S/A - Aperam, Itamarandiba, MG, Brasil

Como citar: Oliveira, L. G. M., Marques, A., Lopes, E. D., Gonçalves, J. F., Martins, N. S., Pena, C. A. A, Arbex, D. C., \& Laia, M. L. (2021). Produtividade, adaptabilidade e estabilidade genotípica de clones de Eucalyptus spp. e Corymbia spp. em diferentes espaçamentos de plantio. Scientia Forestalis, 49(131), e3664.

https://doi.org/10.18671/scifor.v49n131.21

\begin{abstract}
Resumo
Determinados espaçamentos podem causar instabilidade genotípica e gerar perdas de incremento em diâmetro, altura e volume ao longo do tempo. Neste contexto, a análise da interação genótipo $x$ espaçamento (GxE) se torna indispensável. Objetivou-se avaliar a interação (GxE) em um plantio clonal de eucalipto, bem como sua produtividade, adaptabilidade e estabilidade sob diferentes espaçamentos por meio do método da máxima verossimilhança restrita e melhor preditor linear não viesado (REML/BLUP) utilizando as seguintes estratégias: seleção pelo valor genético predito, considerando o desempenho médio dos genótipos entre espaçamentos (sem efeito de interação); considerando o desempenho em cada espaçamento (com efeito da interação); E seleção simultânea quanto à produtividade, adaptabilidade e estabilidade pelo método da média harmônica da performance relativa dos valores genéticos (MHPRVG). O trabalho foi realizado em um plantio clonal com 48 meses de idade implantado em delineamento em blocos ao acaso em arranjo de parcelas subdivididas com seis repetições. Foram avaliados cinco clones de eucalipto (parcelas) e seis espaçamentos de plantio (subparcelas). Foram avaliados os caracteres diâmetro a altura do peito (DAP), altura total (HT) e volume individual com casca (VOL). As estimativas de variância genética obtidas, indicam a possibilidade de expressivos ganhos com seleção. As herdabilidades estimadas foram de alta magnitude associadas a altas acurácias, confirmando a possibilidade de obter ganhos com seleção. Os métodos utilizados permitiram a seleção de clones restritos e clones de utilização ampla. O método MHPRVG proporcionou menores ganhos devido a penalizar a instabilidade genotípica entre os clones.
\end{abstract}

Palavras-chave: Eucalipto; Interação GxE; REML/BLUP; MHPRVG.

\begin{abstract}
Certain spacing can cause genotypic instability and lead to increased losses in diameter, height and volume over time. In this context, genotype $x$ spacing (GXS) interaction analysis becomes indispensable. The objective of this study was to evaluate the interaction (GXS) in a clonal eucalyptus plantation, as well as to evaluate its productivity, adaptability and stability under different spacing using the restricted maximum likelihood method and the best nonlinear linear predictor (REML / Blup) through the following strategies: selection by the predicted genetic value, considering the average performance of the genotypes between spacing (without interaction effect); Considering
\end{abstract}

Fonte de financiamento: Coordenação de Aperfeiçoamento de Pessoal de Nível Superior (CAPES) - Código de Financiamento 001.

Conflito de interesse: Nada a declarar.

Autor correspondente: marcelolaia@gmail.com

Recebido: 20 janeiro 2021.

Aceito: 24 março 2021.

Editor: Paulo Henrique Müller Silva.

(c) Este é um artigo publicado em acesso aberto (Open Access) sob a licença Creative Commons Attribution, que permite uso, distribuição e reprodução em qualquer meio, sem restrições desde que o trabalho original seja corretamente citado. 
the performance in each spacing (with interaction effect); simultaneous selection for productivity, adaptability and stability by the harmonic mean relative performance of genetic values (HMRPGV). The work was carried out in a 48-month-old clonal plantation planted in a block design with six replications, in which six eucalyptus clones (plots) and six planting spacing (subplots) were evaluated. Diameter at breast height (DBH), total height (HT) and individual volume with bark (VOL) were evaluated. The estimates of genetic variance obtained indicate the possibility of significant gains with selection. The estimated heritabilities were of high magnitude and of high accuracy, indicating the possibility of expressive gains with selection. The three methods used permitted the selection of clones for restricted use and clones for wide use. The HMRPGV method provided lesser gains because it penalized the genotypic instability between the spacing.

Keywords: Eucalyptus; GXS interaction; REML/BLUP; HMRPGV.

\section{INTRODUÇÃO}

O espaçamento de plantio refere-se à distância entre as linhas de plantio e entre as plantas nela colocadas. E deve influenciar de forma positiva o crescimento e qualidade da madeira, e proporcionar espaço suficiente para a maximização do crescimento das árvores (Kearney et al., 2007; Magalhães et al., 2007; Silva et al., 2010; Lima et al., 2013). Entretanto, diferentes espaçamentos formam ambientes distintos e segundo Vendruscrolo et al. (2001) podem criar flutuações no desempenho de cultivares. Assim, dificilmente um clone atinge seu máximo desempenho sob diferentes condições de plantio.

Como o ambiente pode afetar a resposta dos materiais genéticos, análises quanto à produtividade, adaptabilidade e estabilidade devido a interação entre genótipo e ambiente (GXA) tornam-se imprescindíveis, por proporcionarem maior confiabilidade quanto a recomendação destes materiais para um determinado ambiente (Rosado et al., 2012; Santos et al., 2016; Teodoro et al., 2016). Autores como Oliveira et al. (2018) reforçam que o conhecimento do efeito do ambiente é fundamental para a seleção de um material genético produtivo. A produtividade enquanto caractere fenotípico resulta da ação do genótipo sob influência do meio, em um determinado ambiente. Ao analisar uma variedade de ambientes, verifica-se, além dos efeitos genéticos e ambientais, o efeito da interação entre os mesmos (Cruz et al., 2012; Borém \& Miranda, 2013). Por consequência, a análise da interação GXA é considerada como uma das etapas de maior importância dentro de um programa de melhoramento e também uma das etapas mais importantes em programas de silvicultura clonal em escala comercial. Pois após a identificação e recomendação dos materiais genéticos que possuem destaque para as características de interesse em diferentes ambientes, é possível realizar o plantio clonal de cada material nos ambientes onde se mostraram mais produtivos (Silva \& Duarte, 2006; Resende, 2007). Assim, tendo em vista à qualidade e produtividade do povoamento o espaçamento apresenta-se, provavelmente, como uma das principais técnicas de manejo.

$\mathrm{Na}$ literatura existem poucos trabalhos recentes envolvendo a interação genótipo $x$ espaçamento (GXE) considerando diferentes espaçamentos como ambientes distintos. Desta forma, destaca-se o trabalho desenvolvido por Valera \& Kageyama (1988), que realizaram um estudo a fim de quantificar a interação GXE em um plantio de Eucalyptus saligna em dois diferentes espaçamentos. De forma similar, Lin et al. (2013) também realizaram um estudo para estimar o efeito da interação GxE em um plantio de Pinus radiata, em três diferentes espaçamentos de plantio. Entretanto, em nenhum dos trabalhos citados, foi avaliado a produtividade, adaptabilidade e estabilidade de forma simultânea e por meio da metodologia de modelos mistos. Diante do exposto e na busca do entendimento do efeito de diferentes espaçamentos no desenvolvimento de clones, o objetivo deste trabalho foi avaliar a interação genótipo x espaçamento (GXE) em um plantio clonal de Eucalyptus spp. e Corymbia spp. bem como realizar seleção genotípica considerando o desempenho médio entre espaçamentos, por espaçamento e pela produtividade, adaptabilidade e estabilidade de forma simultânea entre os espaçamentos testados. 


\section{MATERIAL E MÉTODOS}

O trabalho foi desenvolvido em um plantio experimental, aos 48 meses de idade, pertencente à empresa Aperam Bioenergia S/A, localizada no município de Itamarandiba, região do Vale do Jequitinhonha - MG. A área experimental está localizada nas coordenadas $17^{\circ} 46^{\prime} 22^{\prime \prime} \mathrm{S}$ e $42^{\circ} 54^{\prime} 12^{\prime \prime} \mathrm{W}$, a uma altitude de $946 \mathrm{~m}$.

O clima da região segundo classificação de Koppen é o Cwa, caracterizado como tropical de altitude, temperado úmido, com duas estações bem definidas, verão quente e inverno seco. A região possui uma temperatura média de $21,2^{\circ} \mathrm{C}$, a umidade relativa média do ar varia entre $60 \%$ a $70 \%$ e a média pluviométrica anual histórica é de $1.132 \mathrm{~mm}$ (Instituto Nacional de Meteorologia, 2019). O bioma predominante na região é o Cerrado.

$\mathrm{O}$ experimento foi implantado em delineamento em blocos ao caso em arranjo de parcelas subdivididas com seis repetições, onde as parcelas experimentais foram compostas por plantas de cinco clones de eucalipto (Tabela 1) e as subparcelas foram compostas por seis espaçamentos de plantio (Tabela 2). O plantio foi implantado no mês de setembro de 2014, seguindo os procedimentos silviculturais operacionais da empresa. A subsolagem foi feita em linha com adubação de base utilizando $450 \mathrm{~kg} / \mathrm{ha}$ de NPK (10-20-14) + 1,4\% Ca; 3,0\% S; 0,3\% B; $0,4 \% \mathrm{Cu} ; 0,4 \% \mathrm{Zn}$. O plantio foi realizado de forma manual e a irrigação das mudas foi realizada de maneira mecanizada $\pm 10 \mathrm{~L}^{-1}$ de água por irrigação (3 vezes). 0 replantio das mudas foi realizado 10 dias após o plantio. Aos seis meses de idade foi realizado a calagem em área total com aplicação de $2.000 \mathrm{~kg} / \mathrm{ha}$ de Agrosilício $S^{\circledR}$ e $180 \mathrm{Kg}$ de Magnesita ${ }^{\circledR}$. Também foi realizado o controle de formigas cortadeiras, uma capina química antes e outra oito meses após o plantio e uma adubação de cobertura aplicada na linha de plantio aos 18 meses, utilizando $250 \mathrm{~kg} / \mathrm{ha}$ de NPK (10-00-35) + 0,6\% B + 0,2\% Cu + 0,3\% Zn.

Tabela 1. Material genético utilizado no estudo.

\begin{tabular}{cr}
\hline Clone & Material genético \\
\hline C1 & Eucalyptus cloeziana \\
C2 & Híbrido espontâneo de Corymbia torelliana $\times$ Corymbia citriodora \\
C3 & Híbrido espontâneo de Corymbia citriodora $\times$ Corymbia torelliana \\
C4 & Híbrido espontâneo de Eucalyptus urophylla \\
C5 & Híbrido de polinização controlada de Eucalyptus urophylla $\times($ E. camaldulensis $\times$ E. grandis) \\
\hline
\end{tabular}

Tabela 2. Espaçamentos, densidade de plantas por hectare, número de plantas por parcela e incremento médio anual de clones de Eucalyptus spp. e Corymbia spp. aos 48 meses de idade.

\begin{tabular}{|c|c|c|c|c|}
\hline Clone & Espaçamento & $\begin{array}{c}\text { Densidade } \\
\text { (plantas/ha) }\end{array}$ & $\begin{array}{c}\mathbf{N}^{\circ} \text { de plantas por } \\
\text { parcela }\end{array}$ & IMA $\mathrm{m}^{3} / \mathrm{ha}$ \\
\hline $\mathrm{C} 1$ & $3 \mathrm{~m} \times 3 \mathrm{~m}$ & 1111 & 18 & 17,40 \\
\hline $\mathrm{C} 1$ & $3 \mathrm{~m} \times 1,5 \mathrm{~m}$ & 2222 & 36 & 25,42 \\
\hline $\mathrm{C} 1$ & $3 \mathrm{~m} \times 1 \mathrm{~m}$ & 3333 & 66 & 27,86 \\
\hline $\mathrm{C} 1$ & $6 \mathrm{~m} \times 1,5 \mathrm{~m}$ & 1111 & 18 & 16,79 \\
\hline $\mathrm{C} 1$ & $6 \mathrm{~m} \times 0,75 \mathrm{~m}$ & 2222 & 36 & 21,00 \\
\hline $\mathrm{C} 1$ & $6 \mathrm{~m} \times 0,5 \mathrm{~m}$ & 3333 & 66 & 28,41 \\
\hline $\mathrm{C} 2$ & $3 \mathrm{~m} \times 3 \mathrm{~m}$ & 1111 & 18 & 20,62 \\
\hline $\mathrm{C} 2$ & $3 \mathrm{~m} \times 1,5 \mathrm{~m}$ & 2222 & 36 & 31,75 \\
\hline $\mathrm{C} 2$ & $3 \mathrm{~m} \times 1 \mathrm{~m}$ & 3333 & 66 & 37,36 \\
\hline $\mathrm{C} 2$ & $6 \mathrm{~m} \times 1,5 \mathrm{~m}$ & 1111 & 18 & 21,86 \\
\hline $\mathrm{C} 2$ & $6 \mathrm{~m} \times 0,75 \mathrm{~m}$ & 2222 & 36 & 28,48 \\
\hline $\mathrm{C} 2$ & $6 \mathrm{~m} \times 0,5 \mathrm{~m}$ & 3333 & 66 & 30,15 \\
\hline C3 & $3 \mathrm{~m} \times 3 \mathrm{~m}$ & 1111 & 18 & 34,01 \\
\hline C3 & $3 \mathrm{~m} \times 1,5 \mathrm{~m}$ & 2222 & 36 & 44,71 \\
\hline C3 & $3 \mathrm{~m} \times 1 \mathrm{~m}$ & 3333 & 66 & 44,83 \\
\hline C3 & $6 \mathrm{~m} \times 1,5 \mathrm{~m}$ & 1111 & 18 & 32,62 \\
\hline C3 & $6 \mathrm{~m} \times 0,75 \mathrm{~m}$ & 2222 & 36 & 40,53 \\
\hline C3 & $6 \mathrm{~m} \times 0,5 \mathrm{~m}$ & 3333 & 66 & 38,44 \\
\hline $\mathrm{C} 4$ & $3 \mathrm{~m} \times 3 \mathrm{~m}$ & 1111 & 18 & 33,16 \\
\hline $\mathrm{C} 4$ & $3 \mathrm{~m} \times 1,5 \mathrm{~m}$ & 2222 & 36 & 46,30 \\
\hline $\mathrm{C} 4$ & $3 \mathrm{~m} \times 1 \mathrm{~m}$ & 3333 & 66 & 52,03 \\
\hline $\mathrm{C} 4$ & $6 \mathrm{~m} \times 1,5 \mathrm{~m}$ & 1111 & 18 & 34,52 \\
\hline
\end{tabular}


Tabela 2. Continuação...

\begin{tabular}{ccccc}
\hline Clone & Espaçamento & $\begin{array}{c}\text { Densidade } \\
\text { (plantas/ha) }\end{array}$ & $\begin{array}{c}\mathbf{N}^{\mathbf{0}} \text { de plantas por } \\
\text { parcela }\end{array}$ & ${\text { IMA } \mathbf{~ m}^{\mathbf{3}} / \mathbf{h a}}^{\text {(n) }}$ \\
\hline C4 & $6 \mathrm{~m} \times 0,75 \mathrm{~m}$ & 2222 & 36 & 40,36 \\
C4 & $6 \mathrm{~m} \times 0,5 \mathrm{~m}$ & 3333 & 66 & 44,29 \\
C5 & $3 \mathrm{~m} \times 3 \mathrm{~m}$ & 1111 & 18 & 36,98 \\
C5 & $3 \mathrm{~m} \times 1,5 \mathrm{~m}$ & 2222 & 36 & 50,56 \\
C5 & $3 \mathrm{~m} \times 1 \mathrm{~m}$ & 3333 & 66 & 56,82 \\
C5 & $6 \mathrm{~m} \times 1,5 \mathrm{~m}$ & 1111 & 18 & 39,25 \\
C5 & $6 \mathrm{~m} \times 0,75 \mathrm{~m}$ & 2222 & 36 & 43,54 \\
C5 & $6 \mathrm{~m} \times 0,5 \mathrm{~m}$ & 3333 & 66 & 48,35 \\
\hline
\end{tabular}

Para a mensuração do caráter altura total (HT) foi utilizado um clinômetro digital Haglof ${ }^{\circledR}$. Para a mensuração da circunferência à altura do peito (CAP) foi utilizada uma fita métrica.

As mensurações do CAP foram realizadas em todas as plantas de todas as subparcelas, enquanto as mensurações da altura total foram realizadas sempre medindo uma linha de plantio da subparcela e saltando a linha subsequente.

Após a coleta, os dados foram processados no software Excel (Microsoft $\left.{ }^{\circledR}\right)$, onde os dados de CAP foram transformados em diâmetro a altura do peito (DAP). Para a continuidade das análises dos dados e seleção das melhores árvores, foi seguido o seguinte critério de classificação considerando o estrato ocupado por cada árvore $(H T<(\overline{H T}-1 \sigma))$ "Inferior"; $(\overline{H T}-1 \sigma) \leq H T<(\overline{H T}+1 \sigma)$ "Médio" e $H T \geq(\overline{H T}+1 \sigma)$ "Superior". As árvores classificadas como inferiores, foram retiradas da base de dados.

O volume individual com casca por indivíduo foi calculado a partir dos dados de DAP, HT e fator de forma médio $(0,5244)$.

De posse dos dados de DAP, HT e VOL de todos os indivíduos, foram realizadas as estimativas dos componentes de variância e predição dos valores genéticos por meio da metodologia REML/BLUP e análise simultânea quanto a produtividade, adaptabilidade e estabilidade sob modelos mistos, pelo método MHPRVG utilizando o modelo 55 (adaptado) do software SELEGEN REML/BLUP (Resende, 2002b, 2007). Para tanto, cada espaçamento foi considerado como um ambiente. O modelo utilizado é destinado a experimentos em blocos completos e várias repetições e uma observação por parcela. Assim, o modelo estatístico utilizado foi:

$y=X m+Z g+W p+T i+e$

Onde, $y$ é o vetor de dados, $m$ é o vetor dos efeitos das combinações subparcela-repetição (assumidos como fixos) somados à média geral, $g$ é o vetor dos efeitos de parcela (assumidos como fixos), $p$ é vetor dos efeitos das combinações repetição - parcela (aleatórios), $i$ é o vetor dos efeitos das combinações parcela - subparcela e e é o vetor de erros ou resíduos (aleatórios). As letras maiúsculas representam as matrizes de incidência para os referidos efeitos.

As equações do modelo misto equivalem a:

$$
\left[\begin{array}{cccc}
X^{\prime} X & X^{\prime} Z & X^{\prime} W & X^{\prime} T \\
Z^{\prime} X & Z^{\prime} Z+I^{-1} \lambda_{1} & Z^{\prime} W & Z^{\prime} T \\
W^{\prime} X & W^{\prime} Z & W^{\prime} W+I \lambda_{2} & W^{\prime} T \\
T^{\prime} X & T^{\prime} Z & T^{\prime} W & T^{\prime} T+I \lambda_{3}
\end{array}\right]\left[\begin{array}{c}
\hat{m} \\
\hat{g} \\
\hat{p} \\
\hat{i}
\end{array}\right]=\left[\begin{array}{c}
X^{\prime} y \\
Z^{\prime} y \\
W^{\prime} y \\
T^{\prime} y
\end{array}\right]
$$

Onde,

$\lambda_{1}=\frac{1-\rho}{h^{2}}=\frac{\sigma_{e}^{2}}{\sigma_{g}^{2}} ; \lambda_{2}=\frac{1-\rho}{c^{2}}=\frac{\sigma_{e}^{2}}{\sigma_{c}^{2}} ; \lambda_{3}=\frac{1-\rho}{p^{2}}=\frac{\sigma_{e}^{2}}{\sigma_{p}^{2}}$

$h^{2}=\frac{\sigma_{g}^{2}}{\sigma_{g}^{2}+\sigma_{c}^{2}+\sigma_{p}^{2}+\sigma_{e}^{2}}=$ herdabilidade individual no sentido amplo dentro do bloco; 
$\rho=\frac{\sigma_{g}^{2}+\sigma_{c}^{2}+\sigma_{p}^{2}}{\sigma_{g}^{2}+\sigma_{c}^{2}+\sigma_{p}^{2}+\sigma_{e}^{2}}=$ Repetibilidade individual no bloco;

$P^{2}=\frac{\sigma_{p}^{2}}{\sigma_{g}^{2}+\sigma_{c}^{2}+\sigma_{p}^{2}+\sigma_{e}^{2}}=$ Coeficiente de determinação dos efeitos permanentes da parcela;

$c^{2}=\frac{\sigma_{c}^{2}}{\sigma_{g}^{2}+\sigma_{c}^{2}+\sigma_{p}^{2}+\sigma_{e}^{2}}=$ Correlação ambiental comum entre parcelas.

Os estimadores iterativos dos componentes de variância em REML foram obtidos por meio do algoritmo EM (Expectation-Maximization) (Dempster et al., 1977).

$$
\begin{aligned}
\hat{\sigma}_{e}^{2} & =\left[y^{\prime} y-\widehat{m^{\prime}} X^{\prime} y-\hat{g}^{\prime} Z^{\prime} y-\hat{p}^{\prime} W^{\prime} y-\hat{i}^{\prime} T^{\prime} y\right] /[N-r(X)] \\
\hat{\sigma}_{g}^{2} & =\left[\hat{g}^{\prime} I^{-1} \hat{g}+\hat{\sigma}_{e}^{2} \operatorname{tr}\left(I^{-1} C^{22}\right)\right] / q \\
\hat{\sigma}_{p}^{2} & =\left[\hat{p}^{\prime} p+\hat{\sigma}_{e}^{2} \operatorname{tr} C^{33}\right] / s \\
\hat{\sigma}_{i}^{2} & =\left[\hat{i}^{\prime} i+\hat{\sigma}_{e}^{2} \operatorname{tr} C^{44}\right] / q
\end{aligned}
$$

Em que,

$C=$ Matriz dos coeficientes das equações do modelo misto;

$\operatorname{tr}=$ Operador traço matricial;

$r(X)=$ Posto da matriz $X$;

$N=$ Número total de dados;

$\mathrm{q}=$ Número de indivíduos;

$\mathrm{s}=$ Número de genótipo $\mathrm{x}$ espaçamento.

Os componentes de variância associados aos efeitos do modelo correspondem a:

$\hat{h}_{g}^{2}=\frac{\hat{\sigma}_{g}^{2}}{\hat{\sigma}_{f}^{2}}=$ herdabilidade de parcelas individuais no sentido amplo, ou seja, dos efeitos genotípicos totais;

$\hat{c}_{\text {parc }}^{2}=\frac{\hat{\sigma}_{c}^{2}}{\hat{\sigma}_{f}^{2}}=$ coeficiente de determinação dos efeitos de parcela;

$\hat{c}_{i n t}^{2}=\frac{\hat{\sigma}_{i n t}^{2}}{\hat{\sigma}_{f}^{2}}=$ coeficiente de determinação dos efeitos da interação genótipos x espaçamentos;

$C V_{g}(\%)=\frac{\sqrt{\hat{\sigma}_{g}^{2}}}{\mu} * 100=$ coeficiente de variação genotípica;

$C V_{a}(\%)=\frac{\sqrt{\hat{\sigma}_{c}^{2}}}{\mu} * 100=$ coeficiente de variação residual a;

$C V_{b}(\%)=\frac{\sqrt{\hat{\sigma}_{e}^{2}}}{\mu} * 100=$ coeficiente de variação residual b;

$C V_{r a}=\frac{C V_{g}}{C V_{a}}=$ coeficiente de variação relativa a;

$C V_{r b}=\frac{C V_{g}}{C V_{b}}=$ coeficiente de variação relativa b;

$\hat{r}_{g l o c}=\frac{\hat{\sigma}_{g}^{2}}{\left(\hat{\sigma}_{g}^{2}+\hat{\sigma}_{\text {int }}^{2}\right)}=$ correlação genotípica entre o desempenho nos vários espaçamentos; 
$\hat{h}_{m g}^{2}=\frac{\hat{\sigma}_{g}^{2}}{\hat{\sigma}_{g}^{2}+\left(\hat{\sigma}_{e}^{2} / b\right)+\left(\hat{\sigma}_{c}^{2} /(n b)\right)}=$ herdabilidade da média de genótipo. n: número de parcelas; b: número de blocos;

$\hat{r}_{g g}^{2}=\sqrt{\hat{h}_{m g}^{2}}=$ acurácia na seleção de genótipos.

$\hat{\sigma}_{g}^{2}$ : variância genotípica; $\hat{\sigma}_{c}^{2}$ : variância ambiental entre parcelas; $\hat{\sigma}_{\text {int }}^{2}$ : variância da interação genótipos x espaçamentos; $\hat{\sigma}_{e}^{2}$ : variância residual; $\hat{\sigma}_{f}^{2}$ : variância fenotípica individual; $\mu$ : média geral do experimento.

A seleção simultânea considerando a produtividade, adaptabilidade e estabilidade foi realizada pelo método MHPRVG segundo a expressão $M H P R V G_{i}=n / \sum_{j=1}^{n} * 1 / \sigma_{g_{i^{j}}}^{2}$ em que $n$ é o número de ambientes onde se avaliou o genótipo $i$ e $\sigma_{g_{i j}}^{2}$ é o valor genotípico do genótipo $i$ no ambiente $j$, expresso como proporção da média deste ambiente.

Os efeitos da interação GXA equivalem a interação entre genótipos e ambientes. Desta forma, este efeito foi calculado como sendo o desvio em relação à média geral e livre dos demais efeitos. Além destas análises, a correlação genotípica entre os caracteres DAP, HT e VOL foi obtida por meio do coeficiente de correlação de Pearson (100 iterações) utilizando o pacote psych (Revelle, 2019), disponível para o software R (Core Team, 2019).

\section{RESULTADOS E DISCUSSÃO}

As estimativas dos componentes de variância para os caracteres DAP, HT e VOL são detalhadas na Tabela 3.

Tabela 3. Estimativa dos componentes de variância (REML individual) para os caracteres diâmetro a altura do peito (DAP), altura total (HT) e volume individual (VOL) de clones de Eucalyptus spp. e Corymbia spp. plantados em diferentes espaçamentos.

\begin{tabular}{cccc}
\hline \multicolumn{4}{c}{ Componentes de variância (REML Individual) } \\
\hline Parâmetro & DAP & HT & VOL \\
\hline$\sigma^{2} g$ & 0,43 & 3,64 & 0,0003 \\
erro a & 0,03 & 0,01 & 0,0000 \\
$\sigma^{2}$ int & 0,12 & 0,32 & 0,0001 \\
erro b & 0,15 & 0,13 & 0,0001 \\
$\sigma^{2} f$ & 0,72 & 4,10 & 0,0005 \\
$\mathrm{~h}^{2} g$ & $0,59 \pm 0,16$ & $0,89 \pm 0,20$ & $0,6334 \pm 0,1678$ \\
$c^{2}$ parc & 0,04 & 0,00 & 0,0203 \\
$c^{2}$ int & 0,17 & 0,08 & 0,2319 \\
$\mathrm{~h}^{2} \mathrm{mg}$ & 0,94 & 0,98 & 0,9300 \\
$\hat{r}_{\hat{g} g}^{2}$ & 0,97 & 0,99 & 0,9644 \\
$\hat{r}_{g l o c}$ & 0,78 & 0,92 & 0,7500 \\
$C V_{g}(\%)$ & 6,02 & 12,14 & 21,1200 \\
$C V_{a}(\%)$ & 1,59 & 0,64 & 7,41 \\
$C V_{b}(\%)$ & 0,39 & 2,30 & 12,20 \\
$C V_{r a}(\%)$ & 3,79 & 18,97 & 2,85 \\
$C V_{r b}(\%)$ & 15,43 & 5,27 & 1,73 \\
$\mu$ & 10,90 & 15,71 & 0,0820 \\
\hline
\end{tabular}

$\sigma^{2}$ g: variância genotípica parcela; erro a: erro associado a parcela; $\sigma^{2}$ int: variância da interação parcela x subparcela; erro b: erro associado a subparcela; $\sigma^{2} \mathrm{f}$ : variância fenotípica individual; $h^{2} \mathrm{~g}$ : herdabilidade individual no sentido amplo, ou seja, dos efeitos genotípicos totais; $c^{2}$ parc: coeficiente de determinação dos efeitos de parcela; cint: coeficiente de determinação dos efeitos da interação parcela x subparcela; $h^{2} \mathrm{mg}$ : herdabilidade da média de genótipo; $\hat{r}_{\hat{g} g}^{2}$ : acurácia da seleção de genótipos $\hat{r}_{g l o c}$ : correlação genotípica entre o desempenho nos vários espaçamentos; $C V_{g}(\%)$ : coeficiente de variação genotípica; $C V_{a}(\%)$ : coeficiente de variação residual da parcela; $C V_{b}(\%)$ : coeficiente de variação residual da subparcela; $C V_{r a}$ : coeficiente de variação relativa da parcela; ; $C V_{r b}$ : coeficiente de variação relativa da subparcela $\mu$ : média geral do experimento. 
Como pode-se verificar, as estimativas da variância genotípica $\left(\hat{\sigma}_{g}^{2}\right)$, para o caráter DAP, HT e VOL nos seis diferentes espaçamentos estudados, mostram-se com boas taxas de controle genético sendo 0,48, 3,64 e 0,0003 respectivamente. Estas estimativas evidenciam a existência de variabilidade genética entre os clones e desta forma, uma efetiva seleção quanto a estes caracteres se torna possível. Estimativas dos componentes de variância são de grande importância, pois auxiliam no correto direcionamento do programa de melhoramento, bem como servem de suporte para a correta alocação de materiais genéticos (Maia et al., 2009).

Embora os erros a e b representem 20,00\% para DAP, 3,14\% para HT e 20,00\% para VOL desse valor, significativos progressos genéticos foram obtidos. Rosado et al. (2012) e Torres et al. (2015) ao realizarem análises da interação GXE em eucalipto e feijão-caupi, respectivamente, utilizando modelos mistos, obtiveram variâncias residuais acima de $60 \%$ da variância fenotípica individual e significativos ganhos com seleção.

A proporção desta variância fenotípica que é passível de exploração na seleção, ou seja, a fração que pode ser herdada é denominada de herdabilidade e desta forma, torna-se um dos parâmetros genéticos de maior importância para a seleção. A herdabilidade da média dos genótipos $\left(\hat{h}_{m g}^{2}\right)$ é estimada ao se utilizar as médias como critério de seleção e atinge significativo nível de precisão devido a redução dos erros experimentais por meio do aumento proporcional do número de repetições. Portanto, este componente de variância possui grande importância para os melhoristas, uma vez que para predizer o sucesso do melhoramento na seleção de clones, este parâmetro é essencial, pois estima os valores genotípicos preditos por meio da média de várias repetições (Resende, 2007; Maia et al., 2009; Ramalho et al., 2012; Rosado et al., 2012).

Estimativas de $\hat{h}_{m g}^{2}$ para os caracteres DAP, HT e VOL nos seis espaçamentos apresentaram valores de 0,94, 0,98 e 0,93 respectivamente (Tabela 3), sendo considerados como de alta magnitude. Elevados valores estimados para $\hat{h}_{m g}^{2}$ demonstram que existe alto controle genético na expressão dos caracteres e, consequentemente, grande potencial de seleção dentro do experimento. Estes valores, também servem como indicadores para a seleção de clones de alta produtividade e evidenciam alta precisão experimental (Rosado et al., 2012). Estimativas de $\hat{h}_{m g}^{2}$ classificadas como altas para os caracteres DAP, HT e VOL em clones de Eucalyptus, aos três anos de idade avaliados em quatro ambientes, foram obtidas por Rosado et al. (2012).

A estimativa da herdabilidade individual no sentido amplo $\left(\hat{h}_{g}^{2}\right)$ apresentou-se com valores altos para $\operatorname{DAP}(0,59), \mathrm{HT}(0,89)$ e VOL $(0,63)$, onde Resende $(2002 \mathrm{a})$ a classifica como baixa, de 0,01 a 0,15; moderada, de 0,15 a 0,50 e alta, acima de 0,50. Os valores obtidos para $\hat{h}_{g}^{2}$ demonstram a necessidade de realizar o desdobramento da interação GXE, a fim de maximizar o ganho na seleção dos clones.

Assim, elevadas estimativas do coeficiente de variação genotípica $C_{g}(\%)$ são esperadas, pois ele quantifica a variação genética existente entre os clones para os caracteres avaliados que está disponível para seleção. Portanto, observa-se que os valores obtidos são significativos e que há a possibilidade em obter ganhos genéticos na seleção dos clones. Baixas estimativas para o coeficiente de variação residual $C V_{a}(\%)$ e $C V_{b}(\%)$, também são esperadas, uma vez que esta estatística é um indicativo da qualidade experimental. Segundo Rosado et al. (2012) ao comparar os valores de coeficiente de variação residual entre as variáveis DAP, HT e VOL é esperado que o caráter VOL apresente valores elevados, uma vez que é um caráter obtido indiretamente e desta forma, capitaliza os erros experimentais dos caracteres utilizados ao realizar sua estimativa. Outra variável também conexa a confiabilidade experimental que proporciona uma maior confiabilidade na seleção de clones superiores, é a $\hat{r}_{\tilde{g} g}^{2}$, onde foi-obtido um valor médio de 0,97 para todos os caracteres avaliados. Este valor é considerado como alto e evidencia uma alta correlação entre os valores genéticos preditos e valores genéticos reais dos genótipos (Resende \& Duarte, 2007).

O coeficiente de variação relativa $\left(C V_{r}\right)$ é dado pela relação $C V_{g}(\%) / C V_{a}(\%)$ e $C V_{g}(\%) / C V_{b}(\%)$ possuindo grande relevância em estudos de melhoramento genético, pois fornece uma medida onde é possível observar para um caráter qualquer se o controle genético é maior ou menor e o 
quanto ele é influenciado pelos fatores ambientais, o que permite compreender e/ou detectar a interação GXE para o caráter em análise. Portanto, quanto maior o $\mathrm{CV}_{r}$, maior é o controle genético do caráter e menor é a influência do ambiente sobre ele, valores próximos a 1,00 são considerados como altos e valores médios são considerados quando próximos a 0,5 (Vencovsky \& Barriga, 1992). Os valores de $C V_{r a}$ aqui obtidos foram 3,79, 18,97 e 2,85 para DAP, HT e VOL respectivamente e os valores de $C V_{r b}$ foram 15,43, 5,27 e 1,73 para DAP, HT e VOL respectivamente. Uma vez que, todos foram superiores a 1,00, pode-se inferir que a seleção realizada para estes caracteres serão efetiva do ponto de vista genético e que os diferentes espaçamentos não influenciam de forma acentuada a expressão dos caracteres analisados.

Por sua vez, o coeficiente de determinação dos efeitos de parcela $\left(\hat{c}_{\text {parc }}^{2}\right)$ foi de baixa magnitude para todos os caracteres estudados. Arantes Junior et al. (2013) afirmam que baixos valores de $\hat{c}_{\text {parc }}^{2}$ são desejados, pois indica a qualidade experimental e a homogeneidade ambiental dentro dos blocos instalados.

No que se refere à análise da adaptabilidade e estabilidade genética dos indivíduos, em função do maior ou menor grau desses parâmetros, a variância da interação GxE ( $\left.\hat{\sigma}_{\text {int }}^{2}\right)$ pode inflacionar a expressão fenotípica de um caráter. Esta medida estima o quanto da variação total é representada pela variação da interação GXE (Bastos et al., 2007; Maia et al., 2009). Baixas magnitudes de $\hat{\sigma}_{\text {int }}^{2}$ indicam que a interação GxE emprega pouca influência no valor fenotípico. Neste contexto, um genótipo que demonstre bom desempenho em um ambiente, tende a se manter em ambientes diferentes, pois o genótipo apresentaria uma resposta favorável perante às influências ambientais (alta correlação entre valores genotípicos, através dos ambientes). Desta forma, manteria a constância de seu comportamento ou previsibilidade perante as variações ambientais, ou seja, seria considerado um genótipo estável e, possivelmente, bem-adaptado. Esta estimativa, também permite aferir sobre a necessidade de descriminá-la em estudos de interação, uma vez que a interação GxE possui uma parcela chamada de interação simples e uma chamada de interação complexa ou cruzada (Maia et al., 2009). A interação simples está relacionada à diferença entre a variância genética do caráter em diferentes ambientes e não ocasiona grandes problemas para a seleção. Já a interação complexa é decorrente da ausência de uma correlação entre os tratamentos genéticos de um ambiente para outro. Esta falta de correlação dificulta o processo de seleção de genótipos de adaptação mais ampla (Resende, 2007).

As magnitudes $\hat{\sigma}_{\text {int }}^{2}$ obtidas neste trabalho foram significativas sendo $16,67 \%, 7,81 \% \mathrm{e}$ $20,00 \%$ da variabilidade fenotípica total dos caracteres DAP, HT e VOL, respectivamente. Assim demonstrando que os genótipos estudados, de forma geral, podem apresentar um bom desempenho em diferentes espaçamentos. Comparativamente aos respectivos valores de herdabilidade individual, estes resultados equivalem a 20,34\%, 35,96\% e 0,02\% para DAP, HT e VOL respectivamente, sendo considerados como altos. Estas proporções conduziram a estimativas da correlação genotípica entre o desempenho nos vários ambientes $\left(\hat{r}_{g l o c}\right)$, onde obteve-se valores de 0,78, 0,92 e 0,75 para as variáveis DAP, HT e VOL, respectivamente. Estes resultados corroboram com as estimativas obtidas para o coeficiente de determinação dos efeitos da interação genótipos $x$ ambientes $\left(\hat{c}_{\text {int }}^{2}\right)$, pois segundo Furlan (2018) valores significativos como os estimados, proporcionam elevados valores de $\hat{r}_{\text {gloc }}$. Os valores obtidos para $\hat{r}_{g l o c}$ são considerados altos, entretanto ainda demonstram a necessidade de se analisar a adaptabilidade e estabilidade dos clones nos diferentes ambientes.

Ao analisar a adaptabilidade, estabilidade e produção, mediante emprego das três diferentes estratégias, obteve-se as estimativas de ganhos genéticos preditos para as variáveis analisadas, considerando os valores genotípicos preditos livres de interação $(u+g)$ e os valores preditos somados com a interação média entre os ambientes $(\mathrm{u}+\mathrm{g}+\mathrm{gem})$ de cada clone em todos os ambientes (Tabela 4).

Os valores genotípicos livres da interação permitiram verificar que os três melhores clones foram C3, C4 e C5, com um ganho médio de 5,49\% para DAP, 11,86\% para HT e 18,62\% 
para a variável VOL em todos os espaçamentos e desta forma, podem ser classificados como clones plásticos, ou seja, podem ser utilizados em diferentes espaçamentos e ainda sim obter um desenvolvimento favorável para os caracteres estudados. Esta plasticidade segundo Santos et al. (2013) deve-se, dentre outras razões, ao fato destes clones serem híbridos, pois ao realizar o cruzamento entre espécies nos cruzamentos controlados, isto é, aumentar o número de genótipos que o constitui, maior tende a ser a probabilidade dos genótipos apresentarem estabilidade em diferentes ambientes.

Os significativos valores de ganho quando não se capitaliza a interação média entre os espaçamentos, indicam que é possível recomendar os clones C3, C4 e C5 visando à seleção com base no bom desempenho nos diferentes espaçamentos, ou seja, com base na sua adaptabilidade. Este método penaliza os valores genotípicos preditos, assim o mesmo comportamento das médias genéticas $(u+g)$ dos caracteres analisados é esperado quando os melhores clones forem submetidos a diferentes ambientes, mesmo com padrões de interação GxE diferentes dos encontrados neste estudo. Diferente do observado para estes três clones, os outros dois clones (C1 e C2) apresentaram ganhos considerados como baixos à nulos.

Tabela 4. Estimativas do ganho genético predito (BLUP individual) para os caracteres diâmetro a altura do peito (DAP), altura total (HT) e volume individual (VOL) em clones de Eucalyptus spp. e Corymbia spp. considerando o desempenho médio entre espaçamentos.

\begin{tabular}{|c|c|c|c|c|c|c|c|c|c|}
\hline \multicolumn{10}{|c|}{ DAP } \\
\hline Clone & g & $u+g$ & $\mathbf{G}(\mathbf{u}+\mathrm{g})$ & G \% (u+g) & Nov. Med. & $u+g+g e m$ & $\begin{array}{c}\mathrm{g} \\
(\mathrm{u}+\mathrm{g}+\mathrm{gem})\end{array}$ & $\begin{array}{c}\text { G \% } \\
\text { (u+g+gem) }\end{array}$ & Nov. Med. \\
\hline C3 & 0,78 & 11,68 & 0,78 & 7,11 & 11,68 & 11,71 & 0,76 & 7,01 & 11,66 \\
\hline $\mathrm{C} 4$ & 0,43 & 11,33 & 0,60 & 5,53 & 11,50 & 11,35 & 0,42 & 3,88 & 11,32 \\
\hline $\mathrm{C} 5$ & 0,05 & 10,95 & 0,42 & 3,84 & 11,32 & 10,95 & 0,05 & 0,47 & 10,95 \\
\hline $\mathrm{C} 1$ & $-0,56$ & 10,34 & 0,17 & 1,60 & 11,07 & 10,31 & $-0,55$ & $-5,05$ & 10,35 \\
\hline $\mathrm{C} 2$ & $-0,70$ & 10,20 & 0,00 & 0,00 & 10,90 & 10,17 & $-0,69$ & $-6,32$ & 10,21 \\
\hline \multicolumn{10}{|c|}{ HT } \\
\hline $\mathrm{C} 4$ & 2,53 & 18,24 & 2,53 & 16,10 & 18,24 & 18,30 & 2,55 & 16,22 & 18,25 \\
\hline $\mathrm{C} 5$ & 1,18 & 16,89 & 1,86 & 11,82 & 14,57 & 16,91 & 1,18 & 7,54 & 16,89 \\
\hline C3 & $-0,10$ & 15,61 & 1,20 & 7,66 & 16,91 & 15,61 & $-0,10$ & $-0,64$ & 15,61 \\
\hline $\mathrm{C} 2$ & $-1,74$ & 13,97 & 0,47 & 2,98 & 16,18 & 13,95 & $-1,73$ & $-11,02$ & 13,98 \\
\hline C1 & $-1,87$ & 13,84 & 0,00 & 0,00 & 15,71 & 13,81 & $-1,86$ & $-11,86$ & 13,84 \\
\hline \multicolumn{10}{|c|}{ VOL } \\
\hline $\mathrm{C} 4$ & 0,0186 & 0,1006 & 0,0186 & 22,68 & 0,1006 & 0,1017 & 0,0183 & 22,3427 & 0,1003 \\
\hline $\mathrm{C} 3$ & 0,0114 & 0,0934 & 0,0150 & 18,29 & 0,0970 & 0,0941 & 0,0113 & 13,7232 & 0,0933 \\
\hline C5 & 0,0066 & 0,0887 & 0,0122 & 14,88 & 0,0942 & 0,0891 & 0,0066 & 8,0524 & 0,0886 \\
\hline $\mathrm{C} 1$ & $-0,0177$ & 0,0643 & 0,0047 & 5,73 & 0,0868 & 0,0633 & $-0,0174$ & $-21,2085$ & 0,0646 \\
\hline $\mathrm{C} 2$ & $-0,0189$ & 0,0631 & 0,0000 & 0,00 & 0,0820 & 0,0620 & $-0,0186$ & $-22,6829$ & 0,0634 \\
\hline
\end{tabular}

g: efeitos genotípicos; u + g: valores genotípicos preditos, livres de toda interação com espaçamentos; G: ganho; G \%: ganho em porcentagem; Nov. Med: nova média; u + g + gem: refere-se ao valor genotípico médio nos vários espaçamentos e capitaliza uma interação média com todos os ambientes avaliados.

Entretanto, quando os valores preditos são somados a interação média entre espaçamentos (u+g+gem), apenas os clones C4 e C5 mantiveram-se com bom desempenho para todas os caracteres analisados. Diferente do observado com a abordagem anteriormente considerada, o clone C3 apresentou perda de 0,64\% para o caráter HT, embora tenha apresentado ganhos para as demais variáveis. Desta forma, esse clone foi contabilizado apenas nos ganhos médios para DAP e VOL, onde verificaram-se ganhos médios de 3,78\% para DAP e 14,70\% para VOL. Por sua vez os ganhos médios foram 11,88\% para HT, valor obtido sem contabilizar a contribuição do clone C3. Assim, nesta avaliação os clones C1, C2 e C3 apresentam perdas em relação à média geral do experimento, o que evidenciou a necessidade de analisar seus desempenhos em cada espaçamento.

As Tabelas 5, 6 e 7 demonstram os valores genotípicos preditos ( $u+g+g e$ ) e os ganhos com seleção dos clones em cada espaçamento para os caracteres analisados. De forma geral, os clones C3, C4 e C5, em média, apresentaram ganhos na ordem de 5,81\% quando utilizados no espaçamento $1 ; 6,06 \%$ quando utilizados no espaçamento $2 ; 4,77 \%$ quando utilizados no 
espaçamento 3; 7,77\% quando utilizados no espaçamento $4 ; 5,21 \%$ quando utilizados no espaçamento 5 e 4,64\% quando utilizados no espaçamento 6 para o caráter DAP (Tabela 5). Para o caráter HT os ganhos médios dos clones C3, C4 e C5 foram na ordem de 14,44\% quando utilizados no espaçamento $1 ; 12,70 \%$ quando utilizados no espaçamento $2 ; 10,49 \%$ quando utilizados no espaçamento $3 ; 13,83 \%$ quando utilizados no espaçamento $4 ; 11,74 \%$ quando utilizados no espaçamento 5 e 8,54\% quando utilizados no espaçamento 6 (Tabela 6). Já na Tabela 7, onde são apresentados os ganhos para o caráter VOL, os valores médios obtidos por estes mesmos clones foram de $21,78 \%$ quando utilizados no espaçamento $1 ; 18,92 \%$ quando utilizados no espaçamento $2 ; 18,40 \%$ quando utilizados no espaçamento $3 ; 24,31 \%$ quando utilizados no espaçamento 4; 16,81\% quando utilizados no espaçamento 5 e 14,30\% quando utilizados no espaçamento 6 . Com exceção do clone $C 5$ no espaçamento 6 para os caracteres DAP e VOL os clones C3, C4 e C5 se mantiveram entre os três melhores clones sendo similares aos resultados obtidos e apresentados na Tabela 4 por meio do valor genotípico predito $(\mathrm{u}+\mathrm{g})$ para todos os caracteres em todos os espaçamentos analisados.

Comparativamente, os espaçamentos 3, 5 e 6 proporcionaram ganhos médios inferiores aos ganhos médios observados $(\mathrm{u}+\mathrm{g})($ Tabela 4$)$ para os três melhores clones em todos os caracteres analisados, isto ocorre devido a média do ambiente ser inferior à média do experimento como ocorre no método dos valores genotípicos preditos livres de interação.

Tabela 5. Estimativas do ganho genético predito (BLUP individual) para o caráter diâmetro a altura do peito (DAP) em clones de Eucalyptus spp. e Corymbia spp. considerando o desempenho em cada espaçamento.

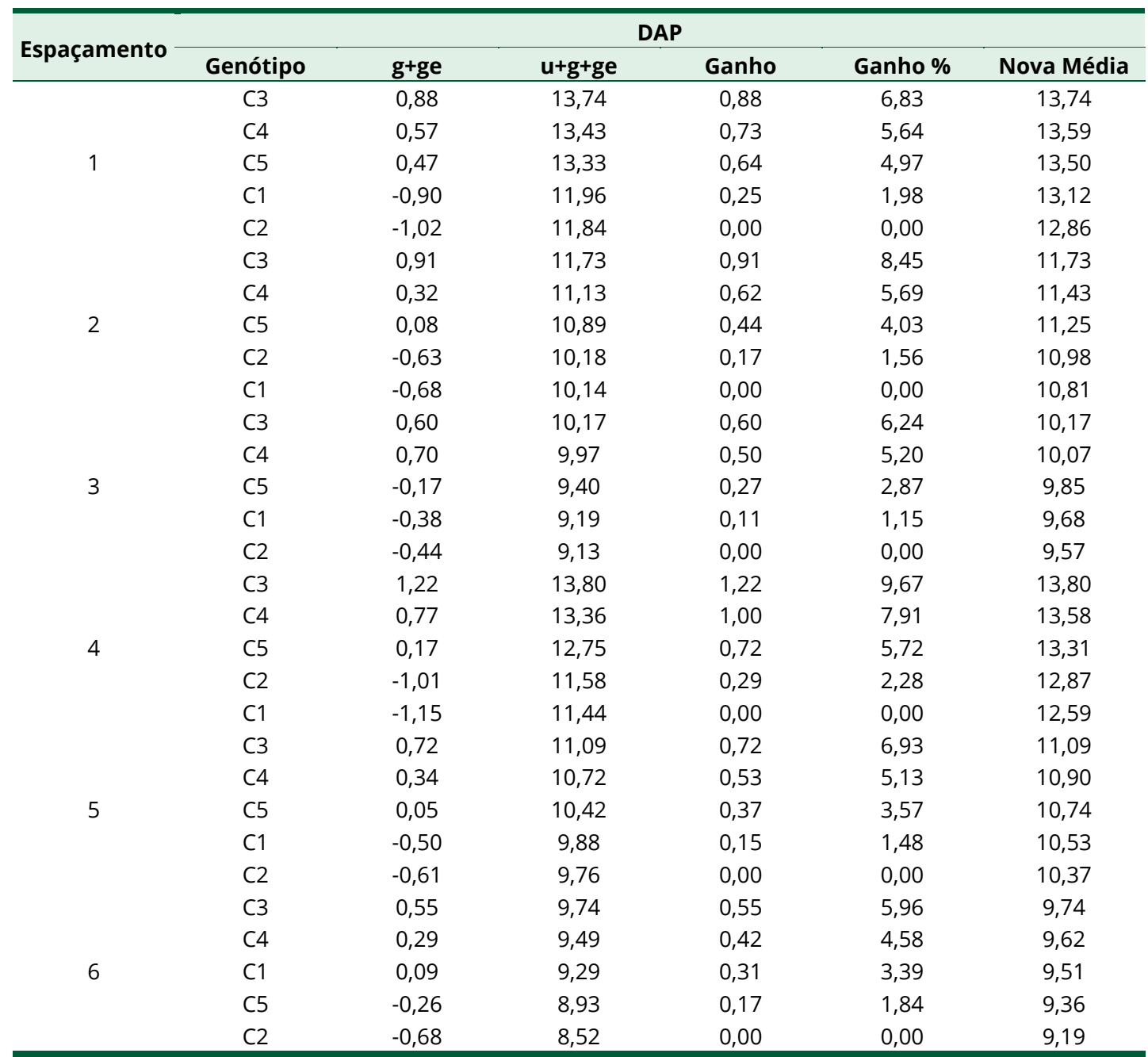

g+ge: efeito genotípico por espaçamento; $u+g+g e:$ valor genotípico predito com capitalização da interação com os espaçamentos. 
Tabela 6. Estimativas do ganho genético predito (BLUP individual) para o caráter altura total (HT) em clones de Eucalyptus spp. e Corymbia spp. considerando o desempenho em cada espaçamento.

\begin{tabular}{|c|c|c|c|c|c|c|}
\hline \multirow{2}{*}{ Espaçamento } & \multicolumn{6}{|c|}{ HT } \\
\hline & Genótipo & g+ge & u+g+ge & Ganho & Ganho \% & Nova Média \\
\hline \multirow{5}{*}{1} & C4 & 3,00 & 19,20 & 3,00 & 18,50 & 19,20 \\
\hline & $\mathrm{C} 5$ & 1,93 & 18,13 & 2,46 & 15,20 & 18,67 \\
\hline & $\mathrm{C} 3$ & $-0,26$ & 15,95 & 1,56 & 9,61 & 17,76 \\
\hline & $\mathrm{C} 2$ & $-2,30$ & 13,91 & 0,59 & 3,66 & 16,80 \\
\hline & $\mathrm{C} 1$ & $-2,37$ & 13,83 & 0,00 & 0,00 & 16,21 \\
\hline \multirow{5}{*}{2} & $\mathrm{C} 4$ & 2,79 & 18,78 & 2,79 & 17,45 & 18,78 \\
\hline & $\mathrm{C} 5$ & 1,22 & 17,20 & 2,00 & 12,53 & 17,99 \\
\hline & $\mathrm{C} 3$ & $-0,11$ & 15,88 & 1,30 & 8,13 & 17,29 \\
\hline & $\mathrm{C} 2$ & $-1,86$ & 14,13 & 0,51 & 3,19 & 16,50 \\
\hline & $\mathrm{C} 1$ & $-2,04$ & 13,94 & 0,00 & 0,00 & 15,99 \\
\hline \multirow{5}{*}{3} & $\mathrm{C} 4$ & 2,42 & 17,86 & 2,42 & 15,67 & 17,86 \\
\hline & C5 & 0,41 & 15,85 & 1,42 & 9,17 & 16,85 \\
\hline & $\mathrm{C} 3$ & 0,24 & 15,67 & 1,02 & 6,62 & 16,46 \\
\hline & $\mathrm{C} 2$ & $-1,51$ & 13,93 & 0,39 & 2,53 & 15,83 \\
\hline & $\mathrm{C} 1$ & $-1,56$ & 13,88 & 0,00 & 0,00 & 15,44 \\
\hline \multirow{5}{*}{4} & $\mathrm{C} 4$ & 3,04 & 19,51 & 3,04 & 18,42 & 19,51 \\
\hline & C5 & 1,60 & 18,07 & 2,32 & 14,06 & 18,79 \\
\hline & $\mathrm{C} 3$ & $-0,18$ & 16,30 & 1,49 & 9,01 & 17,96 \\
\hline & $\mathrm{C} 2$ & $-1,70$ & 14,78 & 0,69 & 4,18 & 17,17 \\
\hline & C1 & $-2,76$ & 13,72 & 0,00 & 0,00 & 16,48 \\
\hline \multirow{5}{*}{5} & $\mathrm{C} 4$ & 2,32 & 17,70 & 2,32 & 15,04 & 17,70 \\
\hline & C5 & 1,52 & 16,91 & 1,92 & 12,46 & 17,31 \\
\hline & C3 & $-0,27$ & 15,12 & 1,19 & 7,73 & 16,58 \\
\hline & $\mathrm{C} 2$ & $-1,66$ & 13,73 & 0,48 & 3,11 & 15,87 \\
\hline & $\mathrm{C} 1$ & $-1,91$ & 13,48 & 0,00 & 0,00 & 15,39 \\
\hline \multirow{5}{*}{6} & $\mathrm{C} 4$ & 1,84 & 16,61 & 1,84 & 12,44 & 16,61 \\
\hline & C5 & 0,52 & 15,30 & 1,18 & 8,00 & 15,95 \\
\hline & C3 & $-0,06$ & 14,71 & 0,77 & 5,19 & 15,54 \\
\hline & $\mathrm{C} 1$ & $-0,74$ & 14,03 & 0,39 & 2,63 & 15,16 \\
\hline & $\mathrm{C} 2$ & $-1,56$ & 13,22 & 0,00 & 0,00 & 14,77 \\
\hline
\end{tabular}

g+ge: efeito genotípico por espaçamento; $u+g+g e:$ valor genotípico predito com capitalização da interação com os espaçamentos.

Tabela 7. Estimativas do ganho genético predito (BLUP individual) para o caráter volume individual (VOL) em clones de Eucalyptus spp. e Corymbia spp. considerando o desempenho em cada espaçamento.

\begin{tabular}{ccccccc}
\hline \multirow{2}{*}{ Espaçamento } & \multicolumn{7}{c}{ VOL } \\
\cline { 2 - 6 } & Genótipo & $\mathbf{g + g e}$ & $\mathbf{u + g + g e}$ & Ganho & Ganho \% & Nova Média \\
\hline \multirow{3}{*}{1} & C4 & 0,0290 & 0,1441 & 0,0290 & 25,20 & 0,1441 \\
& C5 & 0,0205 & 0,1356 & 0,0248 & 21,55 & 0,1398 \\
& C3 & 0,0146 & 0,1296 & 0,0214 & 18,59 & 0,1364 \\
& C1 & $-0,0310$ & 0,0841 & 0,0083 & 7,21 & 0,1233 \\
& C2 & $-0,0331$ & 0,0820 & 0,0000 & 0,00 & 0,1151 \\
2 & C4 & 0,0173 & 0,0966 & 0,0173 & 21,82 & 0,0966 \\
& C3 & 0,0134 & 0,0927 & 0,0154 & 19,42 & 0,0947 \\
& C5 & 0,0063 & 0,0856 & 0,0123 & 15,51 & 0,0916 \\
& C2 & $-0,0180$ & 0,0613 & 0,0048 & 6,05 & 0,0841 \\
& C1 & $-0,0190$ & 0,0603 & 0,0000 & 0,00 & 0,0793 \\
& C4 & 0,0146 & 0,0751 & 0,0146 & 24,13 & 0,0751 \\
& C3 & 0,0085 & 0,0689 & 0,0115 & 19,01 & 0,0720 \\
& C5 & $-0,0010$ & 0,0594 & 0,0073 & 12,07 & 0,0678 \\
& C1 & $-0,0106$ & 0,0499 & 0,0029 & 4,79 & 0,0634 \\
C2 & $-0,0115$ & 0,0490 & 0,0000 & 0,00 & 0,0605 \\
\hline
\end{tabular}


Tabela 7. Continuação...

\begin{tabular}{ccccccc}
\hline \multirow{2}{*}{ Espaçamento } & \multicolumn{7}{c}{ VOL } \\
\cline { 2 - 6 } & Genótipo & g+ge & u+g+ge & Ganho & Ganho \% & Nova Média \\
\hline \multirow{2}{*}{4} & C4 & 0,0331 & 0,1454 & 0,0331 & 29,47 & 0,1454 \\
& C3 & 0,0208 & 0,1330 & 0,0270 & 24,04 & 0,1392 \\
& C5 & 0,0114 & 0,1237 & 0,0218 & 19,41 & 0,134 \\
& C2 & $-0,0284$ & 0,0838 & 0,0092 & 8,19 & 0,1215 \\
& C1 & $-0,0369$ & 0,0753 & 0,0000 & 0,00 & 0,1123 \\
5 & C4 & 0,0144 & 0,0852 & 0,0144 & 20,34 & 0,0852 \\
& C3 & 0,0084 & 0,0792 & 0,0114 & 16,10 & 0,0822 \\
& C5 & 0,0068 & 0,0776 & 0,0099 & 13,98 & 0,0807 \\
& C1 & $-0,0137$ & 0,0571 & 0,0040 & 5,65 & 0,0748 \\
& C2 & $-0,0159$ & 0,0549 & 0,0000 & 0,00 & 0,0708 \\
6 & C4 & 0,0098 & 0,0641 & 0,0098 & 18,05 & 0,0641 \\
& C3 & 0,0070 & 0,0613 & 0,0084 & 15,47 & 0,0627 \\
& C1 & $-0,0014$ & 0,0529 & 0,0051 & 9,39 & 0,0594 \\
& C5 & $-0,0018$ & 0,0526 & 0,0034 & 6,26 & 0,0577 \\
C2 & $-0,0136$ & 0,0408 & 0,0000 & 0,00 & 0,0543
\end{tabular}

g+ge: efeito genotípico por espaçamento; $u+g+g e:$ valor genotípico predito com capitalização da interação com os espaçamentos.

Os três clones (C3, C4 e C5) também se mantiveram entre os três melhores no método onde se capitaliza a produtividade, adaptabilidade e estabilidade (MHPRVG) simultaneamente (Tabela 8). Análise complementar é fundamental para que se tenha maior segurança em estudos de interação GXE (Maia et al., 2009). Pois, a seleção de genótipos estáveis e adaptados aos diferentes ambientes é inerente à capitalização da interação. Entre os três melhores clones para cada ambiente o clone C3 apresentou-se superior aos demais clones analisados quanto ao DAP, seguido dos clones $\mathrm{C} 4$ e C5. Para o caráter HT, houve uma inversão do ranqueamento, assim o clone C4 se mostrou superior aos demais clones, seguido dos clones C5 e C3. E para o caráter VOL o clone C4 também se mostrou superior aos demais clones, seguido dos clones C3 e C5.

Tabela 8. Produtividade, estabilidade e adaptabilidade (MHPRVG e MHPRVG* $\mu$ ) dos valores genotípicos de clones de Eucalyptus spp. e Corymbia spp. para os caracteres diâmetro à altura do peito (DAP), altura total $(\mathrm{HT})$ e volume individual (VOL).

\begin{tabular}{|c|c|c|c|c|c|c|c|c|}
\hline \multicolumn{3}{|c|}{ DAP } & \multicolumn{3}{|c|}{ HT } & \multicolumn{3}{|c|}{ VOL } \\
\hline Clone & MHPRVG & MHPRVG* $\mu$ & Clone & MHPRVG & MHPRVG $^{*} \mu$ & Clone & MHPRVG & MHPRVG* $\mu$ \\
\hline C3 & 1,07 & 11,70 & C4 & 1,16 & 18,26 & C4 & 1,2306 & 0,1010 \\
\hline C4 & 1,04 & 11,34 & C5 & 1,07 & 16,88 & C3 & 1,1442 & 0,0939 \\
\hline C5 & 1,00 & 10,92 & C3 & 0,99 & 15,61 & C5 & 1,0628 & 0,0872 \\
\hline C1 & 0,95 & 10,34 & C2 & 0,89 & 13,95 & C1 & 0,7839 & 0,0643 \\
\hline $\mathrm{C} 2$ & 0,93 & 10,18 & C1 & 0,88 & 13,81 & $\mathrm{C} 2$ & 0,7602 & 0,0624 \\
\hline
\end{tabular}

MHPRVG: média harmônica do desempenho relativo dos valores genotípicos; MHPRVG* $\mu$ : refere-se à MHPRVG multiplicada pela média geral em todos os espaçamentos.

Os clones de maior desempenho quanto a MHPRVG corroboram com os resultados encontrados anteriormente, onde os clones tiveram seu ranqueamento mantido ou não sofreram alterações significativas nos diferentes espaçamentos. A manutenção do ordenamento nas três estratégias de seleção analisadas se deve principalmente pela $\hat{r}_{g l o c}$ obtida para os caracteres DAP, HT e VOL. De posse destes dados, é possível selecionar clones produtivos, adaptados e estáveis que possuam ampla utilização ou que sua utilização é favorável em espaçamentos específicos. Os três melhores clones pelo método MHPRVG, através do valor genético predito (MHPRVG* $\mu$ ), apresentaram ganho médio em relação à média do experimento de 3,63\% para a variável DAP, 7,56\% para HT e 13,65\% para VOL. Ao comparar estes ganhos médios obtidos para os três melhores clones pela seleção em todos os espaçamentos pelo método dos valores genotípicos preditos livre de interação para os caracteres DAP, HT e VOL é observado uma queda de 1,86\%, $4,30 \%$ e $4,97 \%$ respectivamente. E comparando-se estes ganhos médios com os obtidos para a 
seleção por espaçamento (Tabelas 5, 6 e 7) é observado que há uma queda no ganho para todos os clones em todos os espaçamentos.

Porém, ainda que o método MHPRVG proporcione ganhos inferiores aos observados mediante outras analises, este apresenta benefícios adicionais por fornecer resultados da MHPRVG multiplicada pela média geral, que fornece o valor genotípico médio penalizado pela instabilidade e capitalizar a adaptabilidade, tonando-se uma avaliação de grande importância para a recomendação de clones mais adaptados e estáveis.

Estes resultados obtidos demonstram que os clones analisados não interagem de forma complexa com o ambiente, fato este que pode ser comprovado pela manutenção de seus ranqueamentos nos diferentes ambientes, independente da análise considerada. Ao verificar o desempenho dos três melhores clones nos diferentes espaçamentos o ordenamento quanto a superioridade já era esperada, devido aos valores obtidos para $\hat{c}_{i n t}^{2}$ e para $\hat{\sigma}_{\text {int }}^{2}$, o que caracteriza a interação GxE.

Assim, os clones C3, C4 e C5 podem ser recomendados para plantio em outros espaçamentos, desde que respeitado o padrão de interação encontrado neste estudo. Os clones C1 e C2, comparados aos demais clones, apresentaram desempenho inferior em todos os espaçamentos, desta forma, não são recomendados para o plantio nos espaçamentos estudados. Este baixo desempenho está relacionado aos valores negativos encontrados para g + ge, isto é, o genótipo apresenta-se abaixo da média geral do clone no ambiente correspondente.

Os efeitos da interação GxE, são apresentados como desvio em relação à média geral e livre dos demais efeitos tais quais $\mathrm{g}$. A performance de cada clone em cada espaçamento e para cada caractere analisado em suas respectivas grandezas pode ser verificado na Figura 1 por meio das perdas (quando negativo) e ganhos (quando positivos).
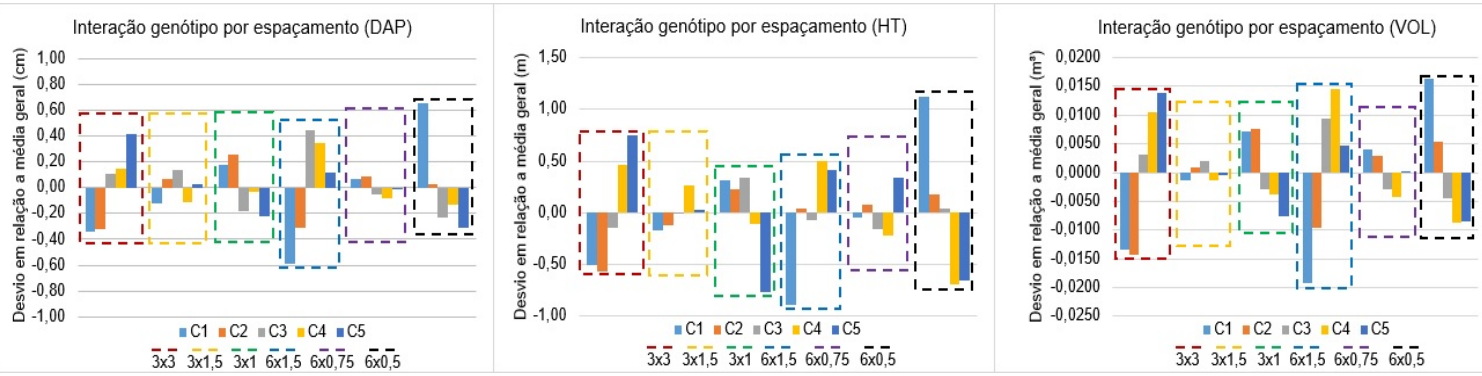

Figura 1. Interação genótipo por espaçamento, para os caracteres diâmetro a altura do peito (DAP), altura total (HT) e volume individual (VOL) para cada clone em cada espaçamento.

A alta correlação genética entre os caracteres DAP, HT e VOL (Tabela 9), demonstra que é possível realizar seleção indireta com vista ao ganho em volume por meio do caráter DAP. Entretanto, para que a seleção indireta seja vantajosa sobre a seleção direta, o produto da acurácia de um caráter quantitativo qualquer pela correlação genética entre ele e o outro caráter quantitativo almejado necessariamente deve ser maior que a acurácia do caráter almejado (Resende, 2002a). Desta forma, a eficiência da seleção indireta foi calculada por meio do caráter DAP visando ganhos com seleção para os caracteres HT e VOL e por meio do caráter HT em vista de ganhos para o caráter VOL (Tabela 9). Entretanto os valores obtidos foram menores que as acurácias dos caracteres aspirados, mesmo obtendo altas correlações entre os mesmos. Assim, neste trabalho a seleção indireta não se torna uma opção vantajosa sobre a seleção direta.

Tabela 9. Eficiência da seleção indireta e correlação genética entre os clones avaliados para os caracteres diâmetro a altura do peito (DAP), altura total (HT) e volume individual (VOL).

\begin{tabular}{cccc}
\hline & DAP & HT & VOL \\
\hline DAP & $\mathbf{1 , 0 0}$ & 0,68 & 0,90 \\
HT & 0,70 & $\mathbf{1 , 0 0}$ & 0,91 \\
VOL & 0,93 & 0,92 & $\mathbf{1 , 0 0}$ \\
\hline
\end{tabular}


A diagonal superior refere à eficiência da seleção indireta e a diagonal inferior refere-se a correlação genética entre os caracteres.

\section{CONCLUSÕES}

A interação genótipo espaçamento é do tipo simples para os clones testados.

As herdabilidades médias dos clones para os três caracteres avaliados, foram de alta magnitude, indicando a possibilidade de se realizar efetiva seleção de clones para os espaçamentos estudados com altas acurácias.

Os três clones com melhor desempenho para os caracteres avaliados nos diferentes espaçamentos foram híbrido espontâneo de Eucalyptus urophylla (C4), híbrido espontâneo de Corymbia citriodora $\times$ Corymbia torelliana (C3) e híbrido de polinização controlada de Eucalyptus urophylla $\times$ (E. camaldulensis $\times$ E. grandis) (C5) respectivamente.

O clone C3 apresentou melhor desempenho para o caractere DAP, quando avaliado simultaneamente em todos os espaçamentos, bem como por espaçamento e quanto a produtividade, adaptabilidade e estabilidade.

O clone C4 apresentou melhor desempenho para os caracteres HT e VOL, quando avaliado simultaneamente em todos os espaçamentos, por espaçamento e para a produtividade, adaptabilidade e estabilidade.

Apenas uma alta correlação genética entre caracteres não permite a efetiva seleção dos clones de forma indireta.

Os clones avaliados apresentaram significativos ganhos com seleção nos espaçamentos utilizados, com exceção dos clones C1 e C2.

O método MHPRVG proporcionou a realização de uma seleção otimizada quanto à produtividade, adaptabilidade e estabilidade, sendo indicado para avaliações e recomendações de clones em diferentes espaçamentos.

\section{AGRADECIMENTOS}

Os autores agradecem a Coordenação de Aperfeiçoamento de Pessoal de Nível Superior (CAPES), o Conselho Nacional de Desenvolvimento Científico e Tecnológico (CNPq), a Fundação de Amparo à Pesquisa do Estado de Minas Gerais (FAPEMIG) e a Aperam Bioenergia S/A, pelo financiamento da pesquisa.

\section{REFERÊNCIAS}

Arantes Junior, F. C., Gonçalves, E. J. S., Moraes, P. S., Gonçalves, M. L. T., \& Resende, E. C. P. (2013). Adaptability and stability in rubber tree progenies under different environmental conditions. Pesquisa Florestal Brasileira, 33(73), 37-44. http://dx.doi.org/10.4336/2013.pfb.33.73.436.

Bastos, I. T., Barbosa, M. H. P., Resende, M. D. V., Peternelii, L. A., Silveira, L. C. I., Donda, L. R., Fortunato, A. A., Costa, P. M. A., \& Figueiredo, I. C. R. (2007). Avaliação da interação genótipo × ambiente em cana-de-açúcar via modelos mistos. Pesquisa Agropecuária Tropical, 37(4), 195-203.

Borém, A., \& Miranda, G. V. (2013). Melhoramento de plantas (6. ed., 523 p.). Viçosa, MG: Ed. UFV.

Core Team. (2019). R: A language and environment for statistical computing. Vienna, Áustria: R Foundation for Statistical Computing. Recuperado em 4 de abril de 2019, de http://R.project.org/

Cruz, C. M., Carneiro, P. C. S., \& Regazz, A. J. (2012). Métodos biométricos aplicados ao melhoramento genético (4. ed., 514 p.). Viçosa: Imp. Universitária.

Dempster, A. P., Laird, N. M., \& Rubin, D. B. (1977). Maximum likelihood from incomplete data via the EM algorithm. Journal of the Royal Statistical Society, 39(1), 1-38.

Furlan, R. A. (2018). Seleção de clones de eucalipto para tolerância à seca no nordeste do Brasil (Tese de doutorado). Universidade Estadual Paulista, Faculdade de Ciências Agronômicas, Botucatu.

Instituto Nacional de Meteorologia - INMET. (2019). Rede de estações automáticas INMET. Recuperado em 5 de fevereiro de 2019, de http://www.inmet.gov.br/portal/index.php?r=home/page\&page=rede_estacoes_auto_graf 
Kearney, D., James, R., Montagu, K., \& Smith, R. G. B. (2007). The effect of initial planting density on branching characteristics of Eucalyptus pilularis and E. grandis. Australian Forestry, 70(4), 262-268. http://dx.doi.org/10.1080/00049158.2007.10675028.

Lima, R., Inoue, M. T., Figueiredo Filho, A., Araújo, A. J., \& Machado, S. A. (2013). Efeito do espaçamento no desenvolvimento volumétrico de Pinus taeda L. Floresta e Ambiente, 20(2), 223-230. http://dx.doi.org/10.4322/floram.2013.001.

Lin, Y., Yang, H., Ivković, M., Gapare, W. J., Colin Matheson, A., \& Wu, H. X. (2013). Effect of genotype by spacing interaction on radiata pine genetic parameters for height and diameter growth. Forest Ecology and Management, 304, 204-211. http://dx.doi.org/10.1016/j.foreco.2013.05.015.

Magalhães, W. M., Macedo, R. L. G., Venturin, N., Higashikawa, E. M., \& Yoshitani Junior, M. (2007). Desempenho silvicultural de clones e espécies/procedências de Eucalyptus na Região Noroeste de Minas Gerais. Revista Ceres, 13(4), 368-375.

Maia, M. C. C., Resende, M. D. V., Paiva, J. R., Cavalcanti, J. J. V., \& Barros, L. M. (2009). Seleção simultânea para produção, adaptabilidade e estabilidade genotípicas em clones de cajueiro, via modelos mistos. Pesquisa Agropecuária Tropical, 39(1), 43-50.

Oliveira, T. W. G., Paula, R. C., Moraes, M. L. T., Alvares, C. A., Miranda, A. C., \& Silva, P. H. M. (2018). Stability and adaptability for wood volume in the selection of Eucalyptus saligna in three environments. Pesquisa Agropecuária Brasileira, 53(5), 611-619. http://dx.doi.org/10.1590/s0100$204 \times 2018000500010$.

Ramalho, M. A. P., Santos, J. B., Pinto, C. A. B. P., Souza, E. A., Gonçalves, F. M. A., \& Souza, J. C. Genética na agropecuária. 5. ed. Lavras: Editora UFLA, 2012, p.566.

Resende, M. D. V. (2002a). Genética biométrica e estatística no melhoramento de plantas perenes (975p.). Brasília: Embrapa Informação Tecnológica.

Resende, M. D. V. (2002b). Software Selegen-REML/BLUP (pp. 67). Colombo: Embrapa Florestas.

Resende, M. D. V. (2007). SELEGEN-REML/BLUP: sistema estatístico e seleção genética computadorizada via modelos lineares mistos (pp. 360). Colombo: Embrapa Florestas.

Resende, M. D. V., \& Duarte, J. B. (2007). Precisão e controle de qualidade em experimentos de avaliação de cultivares. Pesquisa Agropecuária Tropical, 37(3), 182-194.

Revelle, W. (2019). Procedures for Psychological, Psychometric, and Personality: Version 1.8.12. Evanston, Illinois: Northwestern University. Recuperado em 8 de abril de 2019, de https://personalityproject.org/r/psych

Rosado, A. M., Rosado, T. B., Alves, A. A., Laviola, B. G., \& Bhering, L. L. (2012). Seleção simultânea de clones de eucalipto de acordo com produtividade, estabilidade e adaptabilidade. Pesquisa Agropecuária Brasileira, 47(7), 964-971. http://dx.doi.org/10.1590/S0100-204X2012000700013.

Santos, G. A., Nunes, A. C. P., de Resende, M. D. V., Silva, L. D., Higa, A., \& de Assis, T. F. (2016). An index combining volume and Pilodyn penetration to study stability and adaptability of Eucalyptus multispecies hybrids in Rio Grande do Sul, Brazil. Australian Forestry, 79(4), 248-255. http://dx.doi.org/10.1080/00049158.2016.1237253.

Santos, G. A., Resende, M. D. V., Silva, L. D., Higa, A., \& Assis, T. F. (2013). Adaptabilidade de híbridos multiespécies de Eucalyptus ao Estado do Rio Grande do Sul. Revista Árvore, 37(4), 759-769. http://dx.doi.org/10.1590/S0100-67622013000400019.

Silva, E. N., Machado, C. C., Minette, L. J., Souza, A. P., Fernandes, H. C., Silva, M. L., \& Jacovine, L. A. (2010). Avaliação técnica e econômica do corte mecanizado de Pinus sp. com harvester. Revista Árvore, 34(4), 745-753. http://dx.doi.org/10.1590/S0100-67622010000400019.

Silva, W. C. J., \& Duarte, J. B. (2006). Métodos estatísticos para estudo de adaptabilidade e estabilidade fenotípica em soja. Pesquisa Agropecuária Brasileira, 41(1), 23-30. http://dx.doi.org/10.1590/S0100204X2006000100004.

Teodoro, P. E., Bhering, L. L., Costa, R. D., Rocha, R. B., \& Laviola, B. G. (2016). Mixed models for selection of Jatropha progenies with high adaptability and yield stability in Brazilian regions. Genetics and Molecular Research, 15(3), 9. PMid:27706565. http://dx.doi.org/10.4238/gmr.15038824.

Torres, F. E., Teodoro, P. E., Sagrilo, E., Ceccon, G., \& Correa, A. M. (2015). Interação genótipo × ambiente em genótipos de feijão-caupi semiprostrado via modelos mistos. Bragantia, 74(3), 255-260. http://dx.doi.org/10.1590/1678-4499.0099. 
Valera, F. P., \& Kageyama, P. Y. (1988). Interação genótipo x espaçamento em progênies de Eucalyptus saligna Smith. IPEF, 39, 5-16.

Vencovsky, R., \& Barriga, G. P. (1992). Genética biométrica no fitomelhoramento (486 p.). Ribeirão Preto: Sociedade Brasileira de Genética.

Vendruscrolo, E. C. G., Scapim, C. A., Pacheco, C. A. P., Oliveira, V. R., Braccini, A. L., \& Gonçalves-Vidigal, M. C. (2001). Adaptabilidade e estabilidade de cultivares de milho-pipoca na região centro-sul do Brasil. Pesquisa Agropecuária Brasileira, 36(1), 123-130.

Contribuição dos Autores: LGM: Investigação, Metodologia, Escrita - Primeira Redação, Escrita - Revisão e Edição; AM: Escrita - Revisão e Edição; EDL: Conceituação, Metodologia, Escrita - Revisão e Edição; JFG: Escrita Revisão e Edição; NSM: Metodologia, Curadoria de Dados; CAAP: Metodologia, Curadoria de Dados; DCA: Metodologia, Curadoria de Dados; MLL: Conceituação, Análise Formal, Obtenção de Financiamento, Administração do Projeto, Supervisão, Escrita - Revisão e Edição 